ARTÍCULO DE INVESTIGACIÓN

\title{
Anidación del aguililla negra (Buteogallus anthracinus Deppe 1830) en el manglar de Sontecomapan, Catemaco, Veracruz, México
}

\author{
Héctor Hugo Barradas García' \\ Gustavo Carmona Díaz ${ }^{1}$ \\ Ernesto Rodríguez Luna ${ }^{2}$
}

\begin{abstract}
RESUMEN
El aguililla negra (Buteogallus anthracinus) es una especie residente que anida en el manglar de Sontecomapan. El trabajo describe la estructura del nido de $B$. anthracinus, evaluando el tamaño, la composición vegetal de los nidos y la especie de árbol que utiliza para anidar. En 2002 y 2003 mediante recorridos en lancha y con atención a las vocalizaciones emitidas por el adulto ante la presencia de extraños se localizaron seis nidos sobre árboles maduros. Se registró que $B$. anthracinus utiliza a Rhizophora mangle, Laguncularia racemosa y Aviccenia germinans como árboles para establecer sus nidos. Los nidos son ovalados y miden en promedio $38,5 \mathrm{~cm}$ de diámetro interior, $69,83 \mathrm{~cm}$ de diámetro exterior y $52,5 \mathrm{~cm}$ de altura. El centro del nido está construido principalmente de hojas de Avicennia germinans y el resto de Rhizophora mangle. Se discute que la población de $B$. anthracinus puede depender de los sitios de anidación en este manglar.
\end{abstract}

PALABRAS CLAVE:

Anidación, Buteogallus anthracinus, manglar, sitios de anidación.

\begin{abstract}
Buteogallus anthracinus is a tipically resident specie that create its nesting at the mangrove forest of Sontecomapan. This paper describes the nesting of $B$. anthracinus, evaluating size, composition vegetal of the nests and the specie host tree. In 2002 and 2003 by means of routes in boat and with special emphasis to sound emmission to six nests were located on mature trees were. It was found thah Rhizophora mangle, Laguncularia racemosa and Aviccenia germinans are used by $B$. anthracinus as host trees. The nests are oval in shape with an average $38,5 \mathrm{~cm}$ of in diameter, $69,83 \mathrm{~cm}$ of external diameter and $52,5 \mathrm{~cm}$ in height. The center of the nest is constructed mainly withleaves of Avicennia germinans and the rest of Rhizophora mangle. It is discusses that the population of $B$. anthracinus can be dependent on the nest sites in this mangrove.
\end{abstract}

KEY WORDS:

Nesting, Buteogallus anthracinus, mangrove, nest sites. vistas s/n. Xalapa 91000 Veracruz, México. c.e.: hbarradasgarcia@yahoo.com.mx, carmonag@ ecologia.edu.mx.

2 Universidad Veracruzana. Dirección General del Área Académica Biológico-Agropecuarias. Lomas del Estadio S/N. Colonia Zona Universitaria. Xalapa 91000 Veracruz, México. 


\section{INTRODUCCIÓN}

En las aves, la anidación es uno de los aspectos biológicos más relevantes durante su reproducción, así como el periodo cuando son más vulnerables. Los huevos requieren un cuidado y una protección continuos de los padres debido a los depredadores. Por lo tanto, los nidos son construidos en lugares que representen un sitio seguro para el huevo como futura descendencia (Baicich y Harrison, 1997).

Normalmente, las aves rapaces usan el mismo nido por años sólo adicionando material nuevo (Berger 1961; Johnsgard 1990). Otros pueden cambiar de árbol y/o de zona de construcción (Pareja-Obregón 1997; Seipke, 2002; Sánchez y Sánchez, 2002). Se ha registrado que $B$. anthracinus utiliza nuevamente los nidos durante varios años (Schennel, 1994; Maxwell, 1999).

Varios trabajos mencionan algunos aspectos importantes de la anidación de B. anthracinus como: la especie y la estructura de árbol que utiliza para establecer sus nidos, la altura a la cual son construidos y el tamaño de los nidos, sin embargo pocos de estos se han registrado en manglares sin una evaluación de las mismas. Es una especie residente que anida sobre árboles maduros, vigorosos con un promedio de $82 \mathrm{~cm}$ de diámetro, coloca sus nidos desde $2,5 \mathrm{~m}$ a $42 \mathrm{~m}$ sobre el suelo y a $120 \mathrm{~m}$ del agua (Johnsgard, 1990; Schennel, 1994). En promedio los nidos se han encontrado sobre los $15 \mathrm{~m}$ de altura. En pinos (Pinus ponderosa), generalmente entre los $7 \mathrm{~m} \mathrm{a}$ $10 \mathrm{~m}$. En bosque ripario, el árbol sobre el cual se encontró un nido tenía $26 \mathrm{~m}$ de altura con $107,7 \mathrm{~cm}$ de diámetro a la altura del pecho (DAP) y un ancho de dosel de 14,6 m, mismo que reutilizó por dos años seguidos. Se han encontrado nidos en árboles de manglar, sobre especies del género Opuntia, en árbol de algodón Populus spp., en Ficus spp., en cipreses y en otras 14 especies de árboles, utilizando los bordes del bosque para establecer sus nidos y algunas veces estos son reutilizados en años sucesivos (Carter y Wauer, 1965; Johnsgard, 1990; Schennel, 1994; Barrantes, 1998; Maxwell, 1999).

Existe poca información sobre el tamaño y composición vegetal de los nidos de $B$. anthracinus. Los nidos son construidos con ramas, tallos y hojas verdes, estos miden entre $20 \mathrm{~cm}$ y $38 \mathrm{~cm}$ de diámetro en el exterior, $10 \mathrm{~cm}$ en el interior y de $8 \mathrm{~cm}$ a $20 \mathrm{~cm}$ de altura, también se han encontrado nidos de 1,2 $\mathrm{m}$ de diámetro con alturas entre $67 \mathrm{~cm}$ a $12 \mathrm{~m}$ de altura (Schennel, 1994; Baicich y Harrison, 1997; Steinwand, 2000). No obstante, se desconocen las especies vegetales utilizadas para la construcción de los nidos.

En otro aspecto, la población de $B$. anthracinus depende de los sitios de anidación disponibles en el manglar de Sontecomapan (Village, 1983; Newton, 1991). La destrucción de sitios de anidación por la reducción del área vegetal, ha ocasionado la disminución de la densidad de rapaces en estado reproductivo (Newton, 1991; Schennel, 1994). Las aves rapaces son escasas 0 ausentes en áreas donde los sitios de anidación no son los apropiados o los árboles para la formación de nidos no presentan las dimensiones adecuadas requeridas por estas aves (Newton, 1991).

El manglar de Sontecomapan tiene una superficie de 400 hectáreas aproximadamente, está compuesto por tres especies de mangle, Rizophora mangle presente en la orilla, Avicennia germinans y Laguncularia racemosa presentes tierra adentro con dimensiones estructurales altas. La estructura del manglar no es homogénea en toda el área; existen sitios de manglar con individuos de hasta $40 \mathrm{~m}$ 
de altura y cinco metros de DAP que representan sitios disponibles para la reproducción de $B$. anthracinus (Carmona-Díaz y Rodríguez-Luna, 2001).

De ahí que el conocimiento de la anidación de $B$. anthracinus, identificando las características del lugar representen un paso esencial para mantener los hábitats; además son importantes para llevar a cabo estrategias de conservación de esta especie en los diferentes puntos de su distribución (Bisson et al., 2002). La información obtenida de los recursos que necesita $B$. anthracinus para lograr con éxito su anidación, permitirá el establecimiento de nuevas estrategias que lleven a la conservación de esta especie.

\section{OBJETIVOS}

Describir la anidación de Buteogallus anthracinus en el manglar de Sontecomapan, Catemaco, Veracruz. Estimar el tamaño de los nidos de $B$. anthracinus y determinar la composición vegetal de los mismos, así como de los árboles que los soportan.

\section{METODOLOGÍA}

Se llevaron a cabo recorridos en el manglar de Sontecomapan (Fig. 1) durante los meses de enero a mayo del 2002 y 2003, meses que corresponden a la temporada reproductiva de la especie. Durante esta época se hicieron intrusiones en el manglar, principalmente a sitios en que se encontraba el adulto solitario en vuelo o perchado para localizar nidos. Las observaciones se llevaron a cabo por cuatro días consecutivos de cada mes, con binoculares Tasco $10 \times 50$ de las 9:00 h a las 15:00 h, tiempo en que la especie presenta la mayor actividad. Para localizar los nidos se realizaron recorridos lineales (Mikol, 1980) hacia el interior del manglar prestando atención a las vocalizaciones emitidas por el macho o la hembra ante la presencia de extraños cercanos a su nido (Janes, 1985; Mallory y Weatherhead, 1993; Bisson et al., 2002).

Cada árbol con nido fue registrado y marcado sin provocar disturbio (Schennel, 1994; Baich y Harrison, 1997), posteriormente las visitas se llevaron a cabo cada 15 días. Después que el nido era abandonado por la aguililla, se registraron los datos del árbol: diámetro a la altura del pecho (DAP) y cobertura con un flexómetro y altura con la ayuda de un clinómetro Sunto. Para registrar los datos de diámetro interior, exterior y altura, con un flexómetro, se ascendió al nido (Flatten et al., 1989) con un equipo para escalar (arnés, cinturón o bandola, picos y cuerda). Se colectaron ejemplares del material vegetal utilizado en la construcción para ser posteriormente determinado.

\section{RESULTADOS}

Se localizaron en total de seis nidos, dos durante el primer año y cuatro en el segundo, encontrándose que los nidos fueron colocados en los árboles a una altura promedio de $18,32 \mathrm{~m}$, entre las ultimas bifurcaciones de cada árbol. No se encontró preferencia del aguililla para construir los nidos hacia una especie de mangle en particular, ya que los nidos fueron encontrados sobre las tres especies. Sin embargo, tres nidos fueron encontrados sobre Avicennia germinans, dos en Laguncularia racemosa y uno sobre Rhizophora mangle. Los árboles presentaron alturas, DAP y cobertura similares. Con respecto a la distancia con el cuerpo de agua, los nidos fueron encontrados desde los $33 \mathrm{~m}$ hasta los $247,71 \mathrm{~m}$ hacia el interior del manglar, registrándose distancia promedio de 103,39 m (Tabla 1). 


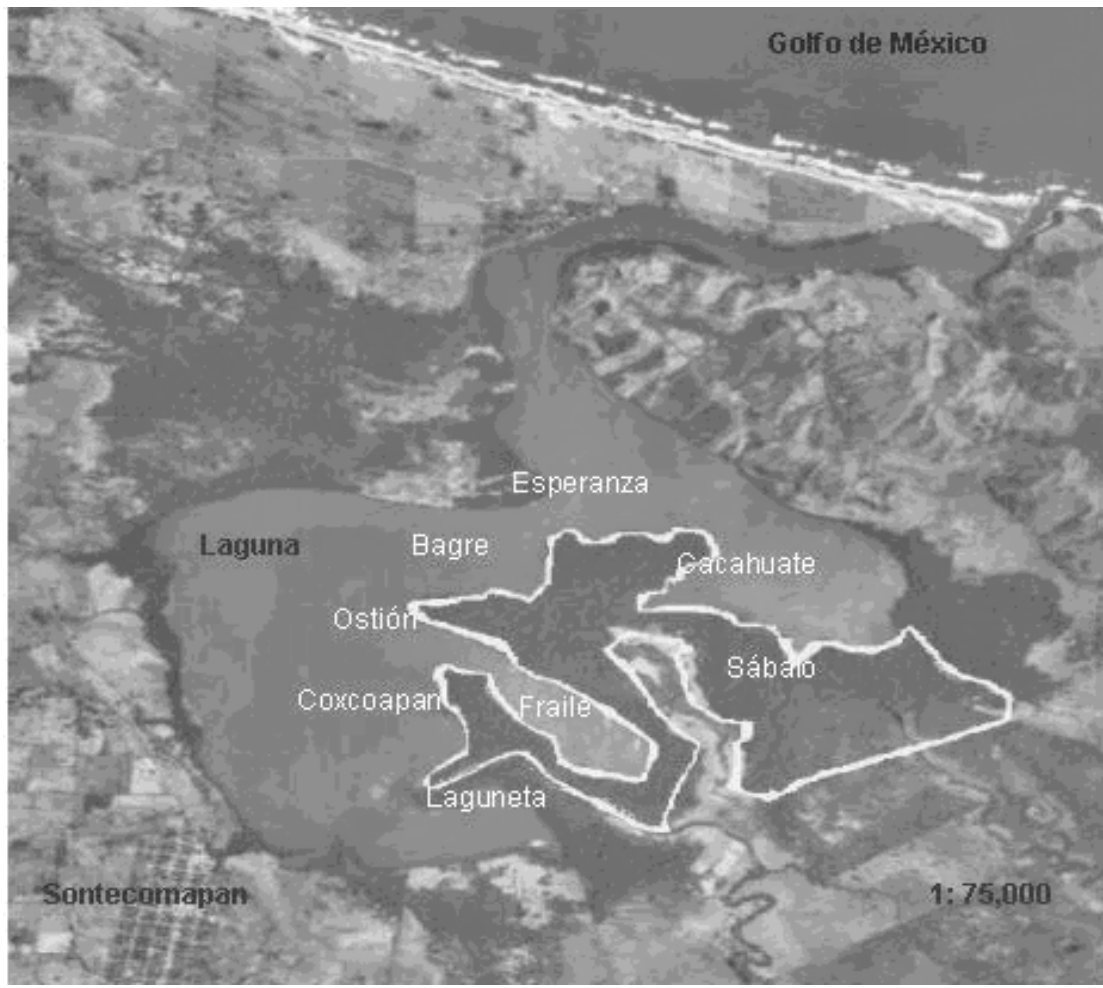

Figura 1. El manglar de Sontecomapan se encuentra cercano al poblado del

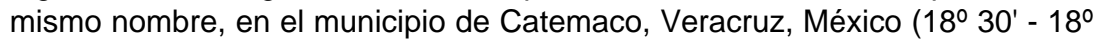
34' norte y 950 00' - 95운 0este), en la región de Los Tuxtlas. Aproximadamente a $14 \mathrm{~km}$ de la ciudad de Catemaco (INEGI, 1998).

Tabla 1. Características de los árboles sobre los cuales fueron encontrados los nidos y la distancia al agua, en el manglar de Sontecomapan, Catemaco, Veracruz

\begin{tabular}{lccccc}
\hline ESPECIE & $\begin{array}{c}\text { ALTURA } \\
(\mathrm{m})\end{array}$ & $\begin{array}{c}\text { DAP } \\
(\mathrm{cm})\end{array}$ & $\begin{array}{c}\text { COBER- } \\
\text { TURA } \\
\left(\mathrm{m}^{2}\right)\end{array}$ & $\begin{array}{c}\text { ALTURA AL } \\
\text { NIDO } \\
(\mathrm{m})\end{array}$ & $\begin{array}{c}\text { DISTANCIA } \\
\text { AL AGUA } \\
(\mathrm{m})\end{array}$ \\
\hline Rhizophora mangle & 23,70 & 42 & 18,85 & 16,70 & 248 \\
\hline Laguncularia racemosa & 36,70 & 60 & 30,32 & 17,20 & 33 \\
\hline Laguncularia racemosa & 31,70 & 93 & 26 & 19,70 & 49 \\
\hline Avicennia germinans & 24,30 & 54 & 25,1 & 19,25 & 66 \\
\hline Avicennia germinans & 35,70 & 72 & 23,4 & 20,20 & 94 \\
\hline Avicennia germinans & 22,90 & 52 & 14,7 & 16,90 & 128 \\
\hline
\end{tabular}


En cuanto a su forma, los nidos fueron ovalados y midieron entre $32 \mathrm{~cm}$ y $46 \mathrm{~cm}$ de diámetro interior, de $67 \mathrm{~cm}$ a 81 $\mathrm{cm}$ de diámetro exterior y presentaron alturas entre los $41 \mathrm{~cm}$ a $61 \mathrm{~cm}$. Fueron construidos con ramas delgadas de las tres especies de mangle y el centro del nido estaba formado principalmente de hojas, que en su mayoría fueron de Avicennia germinans y el resto de Rhizophora mangle. También se encontró que los nidos no son reutilizados, pero la zona de construcción sí resultó ser la misma.

\section{DISCUSIÓN}

Los árboles sobre los cuales fueron encontrados los nidos de $B$. anthracinus en el manglar de Sontecomapan, superan en tamaño a los registrados en otros lugares. Además, la altura promedio sobre la cual fueron encontrados los nidos es mayor a la presentada por Johnsgard (1990) y Schennel (1994) en otros hábitats. Algunos autores mencionan la reutilización de los nidos por esta especie (Schennel, 1994; Maxwell, 1999). Los nidos encontrados el primer año no fueron utilizados al siguiente año, sin embargo, volvieron a anidar sobre árboles cercanos al nido anterior, ocupando la misma "área de anidación".

Uno de los nidos encontrados durante el periodo de incubación fue abandonado conteniendo un huevo. El lugar de anidación es comparable en estructura a los demás, sin embargo difiere de los demás en cuanto a la separación entre los árboles circundantes y el que soporta al nido. Esto permite que el nido sea más visible a los depredadores y/o competidores, característica del lugar que no se tomó en cuenta en el estudio y que podría ser un punto importante para el establecimiento de los sitios de anidación de la especie.
Además, el manglar también es visitado por personas de las comunidades aledañas para la extracción de especies de plantas y animales. Su presencia podría estar afectando con mayor influencia en la temporada de anidación, siendo éste el periodo más critico del ave, provocando así el abandono de los nidos. Por otro lado, en el interior del manglar se observaron varios árboles de grandes tallas que fueron talados, árboles con características que esta especie requiere para llevar a cabo la anidación. El caracterizar los sitios de anidación y el conocimiento de su éxito reproductivo podría sugerir la dirección que deben tomar los esfuerzos de conservación.

Carter y Wauer (1965) y Barrantes (1998) mencionan que $B$. anthracinus utiliza los bordes del bosque para establecer sus nidos, sin embargo, parece ser una característica no muy necesaria para el establecimiento de los nidos esta localidad en ya que en promedio se encontraron a 103,39 m de la orilla hacia el interior del manglar.

Baicich y Harrison (1997) describen el nido de $B$. anthracinus mencionando algunas de sus dimensiones, sin embargo, las medidas que presentan son muy pequeñas en comparación con las presentadas en los nidos del manglar de Sontecomapan, Catemaco, Veracruz (Tabla 2).

\section{CONCLUSIONES}

Buteogallus anthracinus es una especie que requiere árboles maduros de mangle para establecer sus nidos, estos miden en promedio $38,5 \mathrm{~cm}$ de diámetro interior, $69,83 \mathrm{~cm}$ de diámetro exterior y $52,5 \mathrm{~cm}$ de altura, construidos a una altura promedio de 18,32 m. Para llevar a cabo la construcción de los mismos, utiliza largas ramas de las tres especies de mangle y hojarasca para el centro del 
Tabla 2. Datos comparativos de dimensiones de los nidos encontradas en la literatura y las encontradas en el manglar de Sontecomapan

\begin{tabular}{|c|c|c|c|c|}
\hline & \multirow[t]{2}{*}{$\begin{array}{l}\text { DATOS } \\
\text { LITERATURA* } \\
(\mathrm{cm})\end{array}$} & \multicolumn{3}{|c|}{$\begin{array}{c}\text { DATOS MANGLAR DE } \\
\text { SONTECOMAPAN } \\
(\mathrm{cm})\end{array}$} \\
\hline & & PROMEDIO & MÁXIMO & MÍNIMO \\
\hline DIÁMETRO INTERIOR & 20 & 38,5 & 46 & 32 \\
\hline DIÁMETRO EXTERIOR & 10 & 69,8 & 81 & 67 \\
\hline ALTURA & 8 & 52,5 & 61 & 41 \\
\hline
\end{tabular}

nido que en su mayoría proviene de Avicennia germinans.

Es necesario un mayor esfuerzo en la búsqueda de nidos ya que el tamaño de la muestra de este trabajo es pequeño y podría existir alguna preferencia por alguna especie en particular para el establecimiento de los nidos. El tomar en cuenta las características de los árboles circundantes al nido, podría ser de gran importancia para el establecimiento de los mismos. Los sitios de anidación en donde fueron encontrados los nidos mostraban diferencias, en cuanto a la separación del árbol que sostuvo el nido con respecto a los árboles circundantes y la ausencia de árboles maduros podría estar afectando el establecimiento de esta especie.

\section{REFERENCIAS}

Baicich, P.J. y C.J.O. Harrison, 1997. A guide to the nets, eggs, and nestlings of North American birds. Academic Press. EUA. 347 p.

Barrantes, G. 1998. Reproductive activity of birds in a mangrove in Northwest Costa Rica. Universidad de Costa Rica. Heredia, Costa Rica. No. Pág.

Berger, A.J. 1961. Bird study. Dover publications, Inc. Nueva York. EUA. 389 p.
Bisson, A.I.; M. Ferrer y M.D. Bird 2002. Factors influencing nest-site selection by Spanish imperial eagles. J. Field Ornithol. 73(3): 298-302.

Carmona-Diaz, G. y E. Rodríguez-Luna. 2001. Estructura, distribución y abundancia del manglar de Sontecomapan, Catemaco, Veracruz. Memorias del XXI Congreso Mexicano de Botánica. Querétaro, Querétaro. p. 113.

Carter, L.D. y R.H. Wauer.1965. Black hawk nesting in Utah. The condor (67):82-83.

Flatten, C.J.; J.A.M. Madrid; A.E. Hernández y R.P. Gerhardt. 1989. Observaciones del nido de un aguilucho de penacho (Spizaetus ornatus). In: Burnham W.A., J.P. Jenny y C.W. Turley. Reporte de Progreso II, 1989. Proyecto Maya: El uso de aves de rapiña como indicador del medio ambiente para diseño y manejo de áreas protegidas y el desenvolvimiento de capacidad local para la conservación de la naturaleza de América Latina. The Peregrine Fund, Inc. World. p: 85-99

Instituto Nacional de Estadística, Geografía e Informática (INEGI) 
1998. Cuaderno estadístico municipal de Catemaco, Veracruz.

Janes, S.W. 1985. Hábitat selección in raptorial. In: Cody M.L. Habitat and selection in birds. Academic Press, Inc. EUA. p: 159-188.

Johnsgard, A.P. 1990. Hawks, eagles and falcons of North America biology and natural history. Smithsonian Institution Press, Washington y Londres. p: 184-189.

Mallory, L.M. y P.J. Weatherhead. 1993. Observer effects on common goldeneye nest defense. The Condor 95: 467-469.

Maxwell, C.T. 1999. Common black hawk nesting in west-central Texas. J. Raptor Res. 33(3):270-271.

Mikol, S.A. 1980. Field guidelines for using transects to sample nongame bird populations. Performed for western energy and land use team office of biological services. Fish and Wildlife Service. U.S. Department of Interior. Washington D.C. 20240. 27 p.

Newton, I. 1991. Population limitation in birds of prey a comparative approach. In: Perris, C.M., J.D. Lebreton y G.J.M. Irnos. (eds). Bird population studies relevance to conservation and management. Oxford Ornithology Series. Oxford University Press. Oxford, Inglaterra. p: 4-21.

Pareja-Obregón, M.D. 1997. Cetrería y aves de presa "Un duende de nombre Gavilán". Ed. Cairel. España. 368 p.

Ralph, C.J.; G.R. Geupel; P. Pyle; T.E. Martín y D.F. DeSante. 1996. Handbook of field methods for monitoring landbirds. USDA, Forest Service Gen. Tech. Rep. PSW-GTR159-web. p: 41.

Sánchez, E.J. y C. Sánchez-M. 2002. Descripción del nido del gavilán barrado Leucopternis princeps. Conferencia de rapaces Neotropicales. Resúmenes del Simposio sobre el Águila Arpía. The Peregrine Fund. p. 32.

Schennel, H. 1994. Buteogallus anthracinus common Black-hawk. The American Ornithology's Union, EUA. p. 19.

Seipke, H.S. y G.S. Cabanne. 2002. Nidificación del sparvero común (Accipiter erythronemius) en ambientes antropizados en Argentina. Conferencia de rapaces Neotropicales. Resúmenes del Simposio sobre el Águila Arpía. The Peregrine Fund.

Steinwand, J. 2000. Buteogallus anthracinus common Black-hawk. The Regents of the University of Michigan, EUA. p:1-2.

Village, A. 1983. The role of nest-site availability and territorial behavior in limiting the breeding density of Kestrels. Journal of Animal Ecology (52) 2: $635-45 . \diamond$ cinus Deppe 1830) en el manglar de Sontecomapan, Catemaco, Veracruz, México. Madera y Bosques Número especial 2:37-43. 